\title{
High-throughput RNA sequencing from paired lesional- and non-lesional skin reveals major alterations in the psoriasis circRNAome
}

Liviu-lonut Moldovan ${ }^{1,2}$, Thomas Birkballe Hansen ${ }^{1,2}$, Morten Trillingsgaard Ven $\varnothing^{1,2}$, Trine Line Hauge Okholm Thomas Levin Andersen ${ }^{4,5}$, Henrik Hager ${ }^{5}$, Lars Iversen ${ }^{6}$, Jørgen Kjems ${ }^{1,2^{*}}$, Claus Johansen ${ }^{6}$ and Lasse Sommer Kristensen ${ }^{1,2^{*}}$ (i)

\begin{abstract}
Background: Psoriasis is a chronic inflammatory skin disease characterized by hyperproliferation and abnormal differentiation of keratinocytes. It is one of the most prevalent chronic inflammatory skin conditions in adults worldwide, with a considerable negative impact on quality of life. Circular RNAs (circRNAs) are a recently identified type of non-coding RNA with diverse cellular functions related to their exceptional stability. In particular, some circRNAs can bind and regulate microRNAs (miRNAs), a group of RNAs that play a role in the pathogenesis of psoriasis. The aim of this study was to characterize the circRNAome in psoriasis and to assess potential correlations to miRNA expression patterns.

Methods: We used high-throughput RNA-sequencing (RNA-seq), NanoString nCounter technology and RNA chromogenic in situ hybridization (CISH) to profile the circRNA expression in paired lesional and non-lesional psoriatic skin from patients with psoriasis vulgaris. In addition, 799 miRNAs were profiled using NanoString nCounter technology and laser capture microdissection was used to study the dermis and epidermis separately.

Results: We found a substantial down-regulation of circRNA expression in lesional skin compared to non-lesional skin. We observed that this mainly applies to the epidermis by analyzing laser capture microdissected tissues. We also found that the majority of the circRNAs were downregulated independently of their corresponding linear host genes. The observed downregulation of circRNAs in psoriasis was neither due to altered expression levels of factors known to affect circRNA biogenesis, nor because lesional skin contained an increased number of inflammatory cells such as lymphocytes. Finally, we observed that the overall differences in available miRNA binding sites on the circRNAs between lesional and non-lesional skin did not correlate with differences in miRNA expression patterns.

Conclusions: We have performed the first genome-wide circRNA profiling of paired lesional and non-lesional skin from patients with psoriasis and revealed that circRNAs are much less abundant in the lesional samples. Whether this is a cause or a consequence of the disease remains to be revealed, however, we found no evidence that the loss of miRNA binding sites on the circRNAs could explain differences in miRNA expression between lesional and non-lesional skin.
\end{abstract}

Keywords: Psoriasis, Inflammatory diseases, Non-coding RNA, circRNA, microRNA, Genome-wide profiling

\footnotetext{
*Correspondence: jk@mbg.au.dk; lasse@mbg.au.dk

'Department of Molecular Biology and Genetics (MBG), Aarhus University,

DK-8000 Aarhus, Denmark

Full list of author information is available at the end of the article
}

(c) The Author(s). 2019 Open Access This article is distributed under the terms of the Creative Commons Attribution 4.0 International License (http://creativecommons.org/licenses/by/4.0/), which permits unrestricted use, distribution, and reproduction in any medium, provided you give appropriate credit to the original author(s) and the source, provide a link to the Creative Commons license, and indicate if changes were made. The Creative Commons Public Domain Dedication waiver (http://creativecommons.org/publicdomain/zero/1.0/) applies to the data made available in this article, unless otherwise stated. 


\section{Background}

Psoriasis is one of the most common chronic inflammatory skin conditions, with $1-3 \%$ of the adult population affected worldwide [1]. It is defined by a pronounced hyperproliferation and deficient terminal differentiation of the keratinocytes. Moreover, a complex interplay between different cell types (e.g. T cells and dendritic cells) and a variety of cytokines are known to contribute to the development of psoriasis. The pathogenesis is also based on a complex interaction between genetic predisposition, major histocompatibility alleles, and a variety of environmental triggers [2]. From the molecular point of view, however, the mechanisms responsible for the interplay between keratinocytes and inflammatory cells infiltrating the epidermis are still not fully understood. The analysis of the molecular backdrop of psoriasis has described numerous disease-associated genes and proteins with abnormal expression patterns [3], but little is known about the regulatory pathways responsible for this aberrant expression. Recent findings suggest that non-coding RNAs such as microRNAs (miRNAs) and long non-coding RNAs (lncRNAs) could be involved in the pathogenesis of psoriasis by influencing protein expression and function in both keratinocytes and inflammatory cells [4-8].

A new type of non-coding RNA, the circular RNAs (circRNAs), which are formed by a backsplicing event linking the 3' end of an exon to the $5^{\prime}$ end of the same or an upstream exon, have recently been shown to be much more abundant and dynamically expressed than previously appreciated [9-11]. Most circRNAs are expressed from known protein-coding genes and in human cells more unique circRNAs than genes have been annotated [12, 13]. They typically reside in the cytoplasm, are highly stable, evolutionary conserved, and often abnormally expressed in a variety of human diseases, most notably in cancer $[14,15]$. Backsplicing is facilitated by the presence of homologous inverted repeats (e.g. inverted Alu elements (IAE)) in the regions flanking the involved exons [9, 16-19]. These inverted elements can base pair to create a loop structure of the nascent RNA, bringing the splice sites involved in backsplicing into proximity. In support of this biogenesis mechanism, the RNA helicase DHX9 and the RNA editing enzyme ADAR1 were shown to bind double-stranded RNA formed by IAEs and suppress circular RNA production $[16,18,20]$. Conversely, the splicing factors QKI, FUS, and HNRNPL mainly endorse the production of circRNAs [21-23].

The functions of the majority of the annotated circRNAs remain to be disclosed. However, evidence suggests that at least some may function as miRNA sponges [10, 24-28]. Circular RNA sponge for miR-7 (ciRS-7), also known as CDR1as, is the most studied circRNA functioning as a miRNA sponge. It contains more than 70 conserved binding sites for miR-7 [24], but it is still debated whether it acts as an inhibitor, a protector or even as a transporter of miR-7 [29, 30]. Nevertheless, ciRS-7 is not a typical circRNA and the majority do not harbor more miRNA binding sites than expected by chance [31]. Therefore, it is still widely debated whether the ability to act as a miRNA sponge is a general feature of circRNAs [32].

The genome-wide landscape of circRNA expression (the circRNAome) has not previously been revealed in paired lesional- and non-lesional primary skin samples from individuals with psoriasis. Thus, we profiled the circRNAome using high-throughput RNA-sequencing (RNA-seq) and investigated possible mechanisms behind the major differences observed between lesional- and non-lesional skin. We further investigated circRNA expression patterns in individual cell types within the tissues and explored if the dramatic changes observed in the circRNAome have an impact on genome-wide miRNA expression patterns.

\section{Methods}

\section{Patient samples}

Two patient cohorts of Caucasian origin diagnosed with psoriasis vulgaris were recruited for this study. The first cohort consisted of six subjects (average age 42.8 years, range 35-52 years, 1 woman and 5 men). The mean baseline PASI was $16.2(12-20.8)$. PASI for patient 6 was not available. The second cohort consisted of 13 subjects (average age 44.9 years, range 26-62 years, 5 women and 9 men). The mean baseline PASI was 25.6 (17.2-51.8). None of the participants had used any systemic immunosuppressive medications for four weeks and none had received local treatment at the site of biopsies for two weeks before study participation. Four-millimeter punch biopsies were obtained from lesional and nonlesional psoriatic skin taken from the center of a plaque from either the upper or the lower extremities. For each patient, biopsies were taken from only one anatomical site as paired samples and the non-lesional skin at least five centimeters from a plaque. The biopsies were immediately snap-frozen in liquid nitrogen and stored until further use.

\section{Ethical approval}

This study was conducted according to the Declaration of Helsinki Principles. Written informed consent was obtained from each patient and permission from the Regional Ethical Committee of Region Midtjylland, Denmark (M-20090102) and the Danish National Committee on Health Research Ethics was granted (1807975). 


\section{RNA extraction}

Punch biopsies from psoriatic patients were transferred to $1 \mathrm{ml}$ of $-80^{\circ} \mathrm{C}$ cold RNAlater-ICE (Ambion inc., Austin, TX). Samples were kept at $-80^{\circ} \mathrm{C}$ until $24 \mathrm{~h}$ before RNA purification at which time they were transferred to $-20^{\circ} \mathrm{C}$. Upon RNA purification, biopsies were removed from RNAlater-ICE and transferred to $175 \mu \mathrm{l}$ of SV RNA Lysis Buffer added $\beta$-mercaptoethanol (SV Total RNA Isolation System; Promega, Madison, WI) and homogenized. RNA purification, including DNase treatment of the samples, was completed according to the manufacturer's instructions (SV Total RNA Isolation System; Promega, Madison, WI). The RNA was stored at $-80^{\circ} \mathrm{C}$ until further use.

\section{RNA-seq}

RNA from the first cohort, consisting of six patients, was used for RNA-seq analyses. One microgram of total RNA was rRNA depleted using the Ribo-Zero rRNA Removal Kit (Human, Mouse, Rat) (Epicentre, Madison, WI, USA) followed by a purification step using AMPure XP Beads (Beckman Coulter, Brea, CA, USA). Sequencing libraries were generated using the ScriptSeq v2 RNA-Seq Library Preparation Kit (Epicentre) using 12 PCR cycles for amplification. Purification was performed using AMPure XP Beads (Beckman Coulter). The final libraries were size selected (150-500 bp) on a Pippin Prep (Sage Science, Inc. Beverly, MA, USA), quality controlled on the 2100 Bioanalyzer (Agilent Technologies, Santa Clara, CA, USA) and quantified using the KAPA library quantification kit (Kapa Biosystems, Wilmington, MA, USA). RNA-seq was performed on the HiSeq 4000 system (Illumina, San Diego, CA, USA) at the Beijing Genomics Institute (BGI) in Copenhagen using the 100 paired-end sequencing protocol with twelve samples pooled on one lane.

\section{circRNA quantification in RNA-seq data}

Sequencing data from lesional- and non-lesional skin biopsies from the first cohort were quality filtered (Phred score 20) and adapter trimmed using Trim Galore. This resulted in a median of 28,472,184 clean reads (range: $15,893,577-65,463,680)$. Filtered data were mapped to the human genome (hg19) using Bowtie2, mapping only unspliced reads. Unmapped reads were analyzed using a stringent version of the find_circ bioinformatics algorithm [33]. Filtered reads were also mapped to hg19 using Tophat2 and analyzed using CIRCexplorer [34]. All circRNA data analyses were based on the stringent version of the find_circ pipeline and circRNA candidates supported by at least five BSJ (backsplice junction)-spanning reads on average per sample were defined as high abundance circRNAs. Among these, all circRNA candidates not detected by CIRCexplorer were manually inspected to exclude obvious mapping artifacts as previously described [25]. Reads per million (RPM) refers to sequencing reads aligning across the particular BSJ divided by the total number of reads multiplied by one million. The circular-to-linear (CTL) ratios were defined as the number of reads spanning the particular BSJs divided by the average linear reads spanning the splice donor- and splice acceptor sites of the same BSJ plus one pseudo count (to avoid division by zero).

\section{mRNA quantification in RNA-seq data}

Sequencing reads were quality-filtered, and adaptortrimmed as described above. Filtered reads were mapped to hg19 using Tophat 2 and featureCounts [35] was used to quantify the number of reads mapping to annotated genes from Ensembl gene definitions release 71. Differential expression analysis was performed using the DESeq2 $\mathrm{R}$ package, in which the normalization was done using DESeq2's median of ratios method.

\section{Analyses of IAEs}

We used the UCSC Browser RepeatMasker track to extract the distance to nearest IAE flanking the BSJs of the circRNAs analyzed as previously described [36] and by only considering IAEs within the same subfamily (e.g. AluJ or AluS) as previously described [37].

\section{circRNA and gene expression analyses using a custom NanoString nCounter CodeSet}

A custom CodeSet of capture- and reporter probes was designed to target regions of $100 \mathrm{bp}$ overlaying the BSJs of the top 50 most abundant circRNAs in the entire dataset (Additional file 7: Table S1). In addition, seven linear reference genes were included, which have previously been shown to be stable in lesional- and nonlesional skin from patients with psoriasis [38]. We also included capture- and reporter probes designed to target factors that are involved in circRNA biogenesis. These included ADAR, DHX9, FUS, QKI, and HNRNPL. Approximately $150 \mathrm{ng}$ of total RNA from each sample was subjected to nCounter ${ }^{\mathrm{m}}$ SPRINT (NanoString Technologies, Seattle, WA, USA) analysis according to the manufacturer's instructions. The raw data were processed using the nSOLVER 3.0 software (NanoString Technologies); first, a background subtraction was performed using the max of negative controls, and then positive control normalization was performed using the geometric mean of all positive controls. Finally, a second normalization using the geometric mean of the three most stable linear reference genes (ACTB, GUSB and RPL19) was performed, before exporting the data to Excel (Microsoft Corporation, Redmond, WA, USA). These analyses were done on both cohorts. 


\section{miRNA expression analyses}

The nCounter Human v3 miRNA panel (NanoString Technologies), which targets 799 miRNAs, was used for miRNA profiling according to the manufacturer's instructions using $100 \mathrm{ng}$ of total RNA from each sample and a hybridization time of $20 \mathrm{~h}$. The hybridization products were then analyzed on the nCounter ${ }^{\mathrm{rs}}$ SPRINT (NanoString Technologies) platform. The raw data were processed using the nSOLVER 3.0 software (NanoString Technologies); first, a background subtraction was performed using the max of negative controls, and then positive control normalization was performed using the geometric mean of all positive controls. A second normalization was performed using the geometric mean of the best combination of any two miRs (hsa-let-7d-5p and hsa-miR-423-5p) as determined by the NormFinder algorithm [39], before exporting the data to Excel (Microsoft Corporation).

\section{Integrated analysis of miRNA- and circRNA data}

To predict miRNA binding sites within the high abundance circRNAs, we first extracted the expected sequence of the mature circRNAs assuming splicing patterns similar to the host genes by which their share splice sites. Then, for all miRNAs analyzed, we determined the number of 8 mer target sites [40] (an exact match to positions 2-8 of the mature miRNA (the seed + position 8 ) followed by an 'A') present in the circRNA sequences. For each circRNA that was identified as having one or more miRNA binding sites, we calculated the $\triangle \mathrm{RPM}$ values (mean circRNA RPM (lesional) - mean circRNA RPM (non-lesional)). Then, we performed a linear regression analysis of the sum of the $\triangle R P M$ values corresponding to each miRNA and either the fold change of each miRNA (mean miRNA counts (lesional)/mean miR counts (non-lesional)) or the absolute difference in expression level (mean miR counts (lesional) - mean miR counts (non-lesional)).

\section{Microdissections}

After cutting the FFPE punch biopsies into $4 \mu \mathrm{m}$ thick slices, they were mounted on membrane slides (Leica, Germany). The biopsies were deparaffinized for $30 \mathrm{~s}$ in Xylene, rehydrated in a graded ethanol series, stained for $2 \mathrm{~s}$ in hemalun (Mayers Hemalun, Merck, Germany) and washed in sterile water. After drying, 10 consecutive sections from each patient sample (patient 1-5 from the first cohort), epidermis and dermis were microdissected and collected in different tubes using a microdissection system (LMD 630, Leica Germany). RNA purification was done using the miRNeasy FFPE kit (Qiagen, Hilden, Germany).

RNA chromogenic in situ hybridization (CISH)

The abundance of one circRNA, ciRS-7, in 10 sections from the first cohort (patient 1-5) was investigated by
$\mathrm{CISH}$ using a modified version of the RNAScope 2.5 high-definition procedure (310,035, Advanced Cell Diagnostics [ACD], Hayward, CA, USA), as previously described [41]. 3.5- $\mu$ m-thick paraffin sections were pretreated and hybridized with 12 ZZ-pairs (Probe-HsCDR1-AS-No-XMm, 510,711, ACD) targeting ciRS-7 overnight. The ZZ-pairs binding ciRS-7 were detected using seven amplification steps, including a Tyramide Signal Amplification step (TSA-DIG; NEL748001KT, PerkinElmer, Skovlunde, Denmark) labeled with alkaline phosphatase-conjugated sheep anti-DIG FAB fragments $(11,093,274,910$, Roche, Basel, Switzerland), before visualized with Liquid Permanent Red (DAKO, Glostrup, Denmark) and counterstained with Mayer's hematoxylin.

\section{Statistical analyses}

All statistical tests were performed using Prism 7 (GraphPad, La Jolla, CA, USA). Comparisons of the average expression levels of the high abundance circRNAs, as well as miRNAs, between the lesional- and non-lesional skin were done using Wilcoxon matchedpairs signed rank tests, as the data were not normally distributed according to the D'Agostino \& Pearson normality test. Volcano plots were generated by one unpaired $t$ test per circRNA individually without assuming consistent standard deviation and without correction for multiple testing. Linear regression was used to assess the potential correlation between fold changes in RPM and fold changes in CTL ratios employing an F test to investigate if the slope was significantly non-zero. Assessment of differences in expression levels of $A D A R, Q K I, F U S$, $D H X 9$, and HNRNPL as well as T-cell-specific genes between the lesional- and non-lesional skin was done using paired $\mathrm{t}$ tests. To assess whether circRNAs downregulated > two fold in lesional skin were more likely to have Alu-mediated biogenesis relative to the remaining circRNAs, Chi-squared tests were employed. Mann Whitney tests were used to assess whether the distances to the nearest IAE flanking the BSJs were different between circRNAs downregulated $>$ two fold in lesional skin relative to the remaining circRNAs. Analyses of circRNAand mRNA expression in the microdissected samples were done using unpaired t tests, as data were not available for all sample pairs. The tests were done with Welch's correction when variances were significantly different as determined by an F test. All $P$-values were twotailed and considered significant if $<0.05$.

\section{Results}

Identification and characterization of circular RNAs in lesional- and non-lesional skin biopsies

In order to perform an unbiased genome-wide profiling of circRNAs in psoriasis, we performed RNA-seq of ribosomal RNA-depleted total RNA from paired lesional- $(n=6)$ 
and non-lesional $(n=6)$ skin biopsies. We detected 2066 and 2626 unique circRNAs supported by at least two BSJspanning reads in a single sample in the lesional- and non-lesional skin biopsies, respectively, using a stringent version of the find_circ bioinformatics algorithm [33]. However, to ensure that we are looking at bona fide circRNAs with reasonable expression levels, the following analyses only included circRNAs supported by an average of at least five BSJ-spanning reads in each sample type. This resulted in 128 and 285 circRNAs (defined as high abundance circRNAs) in the lesional- and non-lesional skin, respectively. Most of these, 117 of 128 (91.4\%) and 261 of 285 (91.6\%), were also detected by the CIRCexplorer bioinformatics algorithm that only considers circRNAs derived from annotated splice sites [34]. Forty-one (32.0\%) and seventy-three (25.6\%) circRNAs were on average expressed at higher levels than their linear host genes (circular-to-linear (CTL) ratio $>1$ ), and we detected four and two potentially novel circRNAs (not present in circBase [12] or CIRCpedia v2 [13]) in the lesional- and the non-lesional skin, respectively (Additional file 7: Tables S2 and S3). Among the top 50 most abundant circRNAs in each sample type, we found four circRNAs present in both tissue types that have previously been shown to be highly expressed in several different tissues in RNA-seq data from the ENCODE consortium (known as the top 10 alpha circRNAs) [36] (Fig. 1a and b). The 128 and 285 circRNAs were generated from 115 and 241 different host genes, respectively (Fig. 1c and d). Finally, we observed that circRNAs composed of two exons were most frequent in both sample types (Fig. 1e and f).

\section{circRNAs are less abundant in lesional psoriatic skin relative to non-lesional skin}

In total, we detected 298 unique high-abundance circRNAs in the lesional- and non-lesional skin biopsies combined. The overlap between circRNAs detected in the lesional- and non-lesional skin was considerable (38.6\%), but many were unique mainly to the nonlesional skin (Fig. 2a). In line with this, we observed that the 298 circRNAs were generally present at lower levels in lesional skin (Fig. 2b). This was also true when comparing the lesional- and non-lesional skin for each individual patient (Additional file 1: Figure S1). In total, 148 circRNAs, including ciRS-7 (CDR1as), were significantly downregulated in lesional skin, while none was significantly upregulated (Fig. 2c and Additional file 7: Table S4). To investigate if the dramatic downregulation of circRNAs in lesional skin was due to reduced expression of their host genes, fold changes in RPM were plotted against fold changes in CTL ratios. We observed that most of the circRNAs appeared along a diagonal line, indicating that they were downregulated independently of their host genes. However, there was also a substantial number of the downregulated circRNAs for which their respective host genes were downregulated to some extent (represented by dots appearing above the diagonal line in Fig. 2d). In addition, we did not observe a considerable downregulation in lesional skin relative to nonlesional skin when considering other classes of RNA (Additional file 2: Figure S2).

Next, we wanted to rule out whether the observed downregulation was caused by experimental bias. Template switching and rolling circle amplification during reverse-transcription (RT) are major concerns in the circRNA research field as artifacts mimicking circRNA molecules may arise because of these phenomena [42-44]. Therefore, we employed an enzyme-free technology (termed NanoString nCounter) [43] to profile the expression of the top 50 most abundant circRNAs in the entire dataset within the same samples. Again, we observed that circRNAs are dramatically downregulated in the lesionalrelative to non-lesional skin (Additional file 3: Figure $\mathrm{S} 3 \mathrm{~A}$ ). To extend the generality of the observed downregulation of circRNAs in psoriasis, we further analyzed six normal skin samples from unaffected individuals using our NanoString nCounter panel. Again, we observed a profound downregulation of circRNAs when comparing this data to data obtained from lesional skin (Additional file 3: Figure S3B). Finally, we analyzed a second cohort consisting of paired lesional- and non-lesional skin samples from another 13 psoriasis patients using our NanoString nCounter panel and confirmed the previous observations that circRNAs are generally less abundant in lesional skin (Additional file 3: Figure S3C).

\section{Factors known to influence circRNA biogenesis are unlikely to be the main drivers of circRNA downregulation in lesional skin}

Several RNA-binding proteins, encoded by $A D A R$, FUS, $D H X$ 9, HNRNPL, and QKI have previously been shown to regulate circRNA biogenesis [16, 20-23]. Thus, we speculated that expression changes of these genes might explain the observed downregulation of circRNAs in lesional skin. Interestingly, $A D A R$ was significantly upregulated in lesional skin both when considering the RNA-seq data (Fig. 3a) and the NanoString nCounter data in both psoriasis cohorts (Fig. 3b and Additional file 4: Figure S4). Because $A D A R$ is known to suppress circRNA biogenesis mediated by base pairing between IAEs flanking the BSJs [16], we searched for IAEs within 2300 nucleotide regions flanking the BSJs (total distance) as previously described [16] for each of the high abundance circRNAs. The fraction of circRNAs with neighboring IAEs was 44\% (131/ 298) and there was no tendency for circRNAs with IAEs to be more downregulated than circRNAs without them $(P=0.75$, chi-squared test) (Fig. 3c). Consistent with this, the median distance to the nearest IAE did not differ 


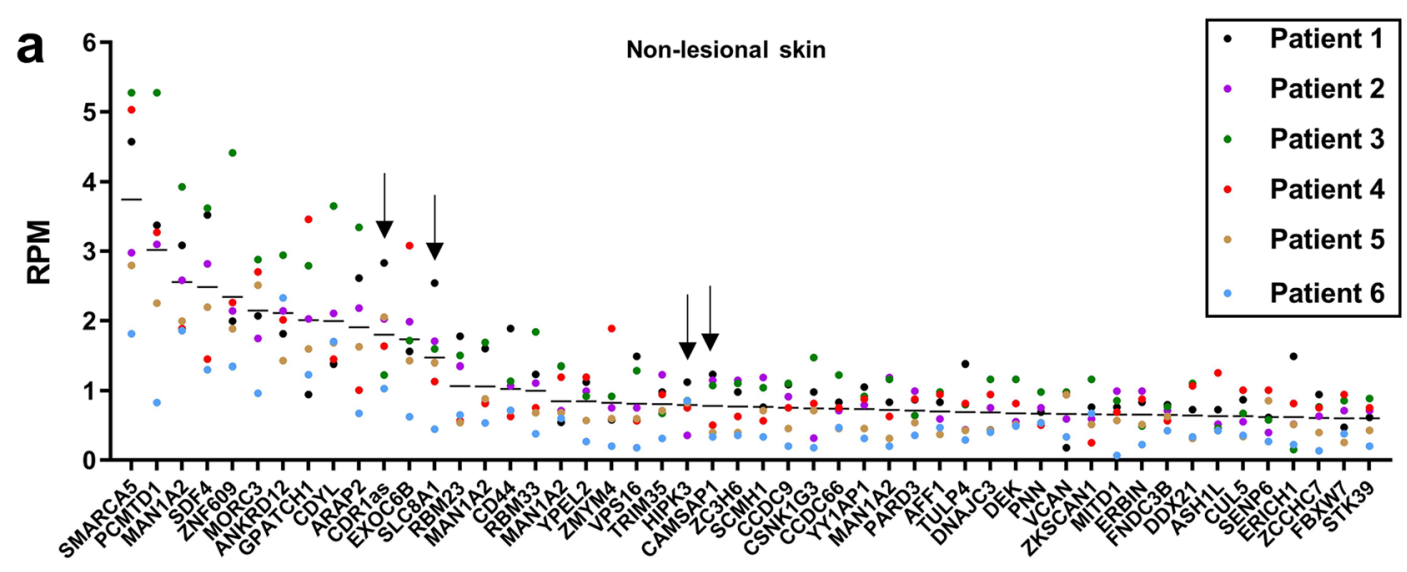

b
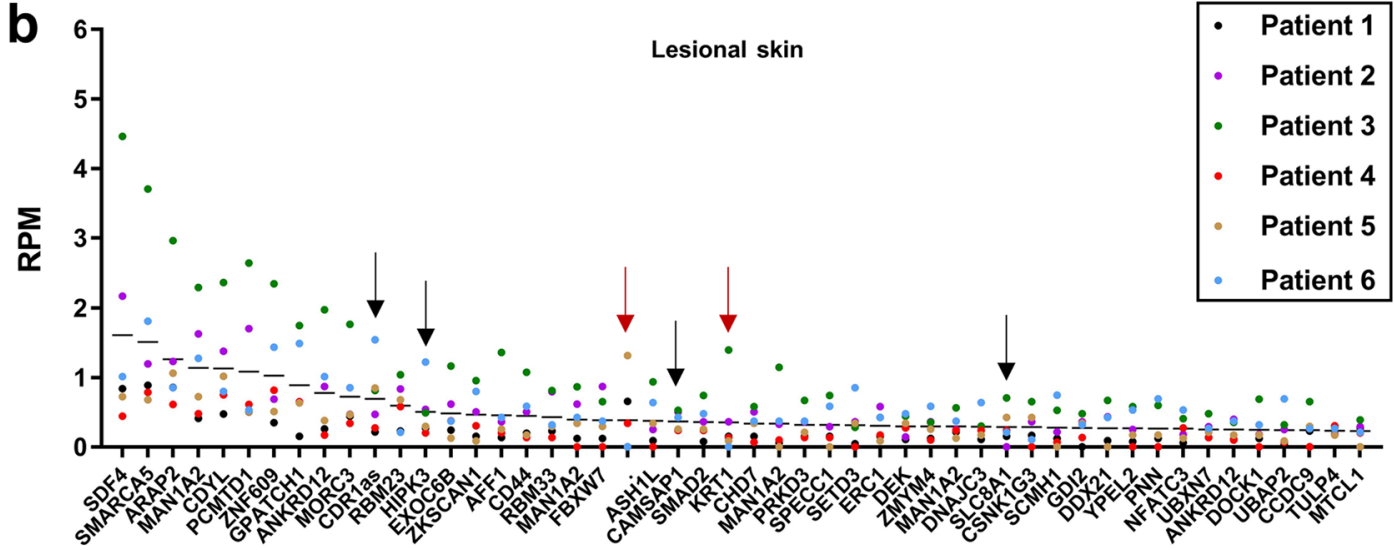

C

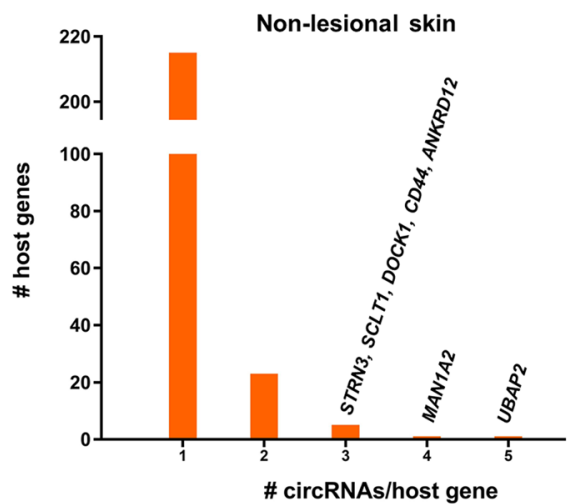

e

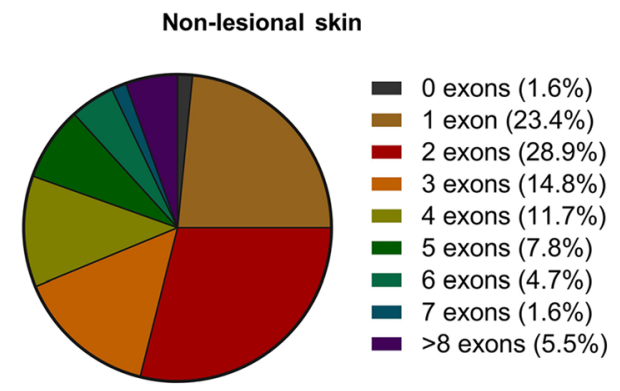

d

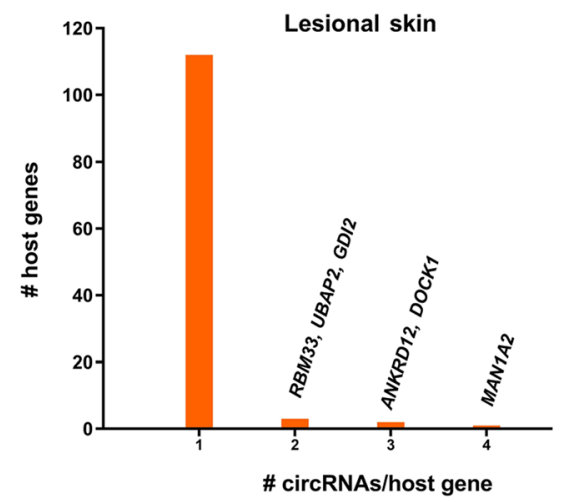

f

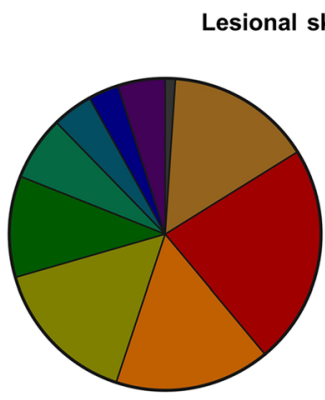

- 0 exons $(1.1 \%)$

- 1 exon $(15.1 \%)$

- 2 exons $(22.8 \%)$

- 3 exons $(16.1 \%)$

- 4 exons $(15.4 \%)$

- 5 exons $(10.5 \%)$

- 6 exons $(6.7 \%)$

- 7 exons $(4.2 \%)$

- 8 exons $(3.2 \%)$

- >8 exons $(4.9 \%)$

Fig. 1 (See legend on next page.) 
(See figure on previous page.)

Fig. 1 Characterization of circular RNAs in lesional- and non-lesional skin using RNA-seq. (a-b) Expression levels of the top 50 most abundant circRNAs in non-lesional skin (a) and lesional skin (b). Each dot represents the expression in reads per million (RPM) for a circRNA in one individual sample represented by a unique color. Each line represents the mean RPM. Black arrows indicate top 10 alpha circRNAs and red arrows indicate circRNAs not present in circBase. (c-d) Histograms showing the number of host genes producing various numbers of unique high abundance circRNAs in non-lesional skin (c) and in lesional skin (d). (e-f) Pie charts showing the distribution of the numbers of exons annotated within the high abundance circRNAs in non-lesional skin (e) and lesional skin (f)

significantly between circRNAs downregulated more than two-fold and the remaining circRNAs $(P=0.10$, Mann Whitney test). In addition, we searched for IAEs within 10,000 nucleotide regions flanking the BSJs (total distance), this time requiring them to belong to the same subfamily as previously described in another publication [37]. Again, there was no tendency for circRNAs with IAEs from the same subfamilies to be more downregulated than circRNAs without $(P=0.60$, chi-squared test) (Additional file 5: Figure S5A), and also the median distance to the nearest IAE did not differ significantly between circRNAs downregulated more than two fold and the remaining circRNAs $(P=0.13$, Mann Whitney test). Together, these analyses imply that other mechanisms are likely to be responsible for the observed downregulation of circRNAs in lesional skin.

\section{circRNA expression in different cellular compartments of the skin biopsies}

It is well known that lesional psoriatic skin contains more inflammatory cells, such as lymphocytes, than non-lesional skin $[45,46]$. If the lymphocytes were to contain fewer circRNAs than keratinocytes, this could be a plausible explanation for the observed downregulation of the circRNAome in lesional skin. Before testing this hypothesis, we first confirmed that our lesional skin biopsies contained more lymphocytes than non-lesional skin. From hematoxylin and eosin staining of fixed skin biopsy sections, we found that especially the dermis of lesional skin showed greater lymphocytes numbers relative to the dermis of the non-lesional skin (Fig. 4a). By analyzing the expression levels of $\mathrm{T}$-cell specific genes [47], we could also confirm that the lesional skin contained more lymphocytes. In particular, four of six T-cell specific genes analyzed were significantly upregulated in the lesional skin (Fig. 4b). Then, because the majority of the lymphocytes reside in the dermis, we separated the dermis from the epidermis by laser capture microdissection of five lesional skin- and five non-lesional skin biopsies and profiled the expression of the top 50 most abundant circRNAs using NanoString nCounter technology. Due to limited amounts of RNA, only seven circRNAs reached the detection limit in at least half of the samples. When comparing lesional and non-lesional epidermis, five of the seven circRNAs, including ciRS-7, were significantly downregulated in the lesional skin, whilst the analysis of the dermis showed only one circRNA to be significantly different between the two tissue types (Fig. 4c and d). In line with the above-mentioned analysis of factors known to affect circRNA biogenesis, we observed an upregulation of $A D A R$ in lesional skin. This applied to both the dermis and the epidermis (Fig. $4 \mathrm{e}$ and $\mathrm{f}$ ), supporting the notion that $A D A R$ is not instrumental for the downregulation of the circRNAome in lesional skin.

Next, to back our findings that circRNAs are mainly downregulated in the epidermis, we performed RNA chromogenic in situ hybridization (CISH) for ciRS-7, since this circRNA was significantly downregulated in both the RNA-seq data and in the NanoString nCounter data for both cohorts. Consistent with these analyses, we observed a marked downregulation of ciRS-7 in the epidermis of lesional- relative to nonlesional skin (Fig. 5).

\section{Identification and characterization of miRNAs in lesional- and non-lesional skin biopsies}

Having established that circRNAs are profoundly downregulated in lesional skin, we speculated that this phenomenon could be responsible for previously reported alterations in miRNA expression associated with psoriasis $[4,5]$. To investigate this, we profiled the expression of approximately 800 miRNAs using the nCounter Human v3 miRNA panel from NanoString Technologies, in lesional and non-lesional skin.

We detected 182 and 143 unique miRNAs supported by at least two counts in a single sample in the lesional $(n=6)$ and non-lesional skin biopsies $(n=6)$, respectively. To ensure that we look at miRNAs with reasonable expression levels, we only considered miRNAs supported by an average of at least five counts (defined as high abundance miRNAs) in each sample type. This resulted in a total of 123 and 106 miRNAs in the lesional skin- and non-lesional skin biopsies, respectively.

Combining the lesional- and non-lesional skin samples, we detected 137 unique high abundance miRNAs, with a substantial overlap between the miRNAs detected in each sample type (67.2\%) (Fig. 6a). When considering all patients combined, the median expression of the high abundance miRNAs was significantly higher in lesionalrelative to non-lesional skin (Fig. 6b). However, this did 


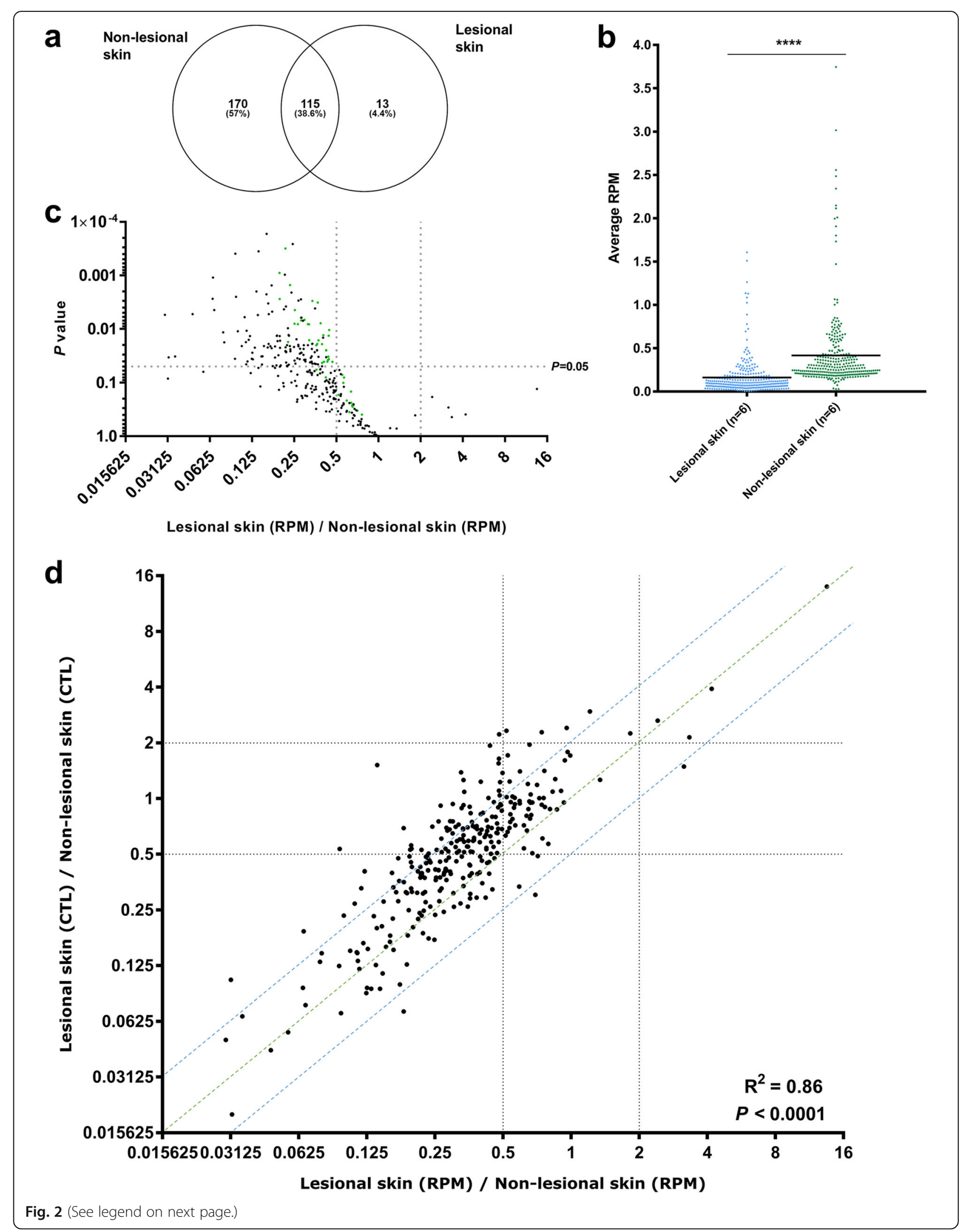


(See figure on previous page.)

Fig. 2 The circRNAome is massively downregulated in lesional- relative to non-lesional skin. (a) Venn diagram illustrating the overlap of circRNAs detected in the lesional- and the non-lesional skin. (b) Scatter plot showing that the average expression in reads per million (RPM) of the 298 unique high-abundance circRNAs is lower in lesional- relative to non-lesional skin. (c) Volcano plot of the 298 unique high-abundance circRNAs showing fold changes in circRNA expression in RPM between lesional- and non-lesional skin according to the levels of significance. The top 50 most abundant circRNAs are indicated in green. (d) Scatter plot of fold changes in RPM and fold changes in circular-to-linear (CTL) ratios of the high abundance circRNAs. It can be observed that most circRNAs were downregulated independently of their respective host genes (defined as those being present in between the blue lines)

not apply to each individual patient (Additional file 6: Figure S6). In total, 37 miRNAs were differentially expressed; 31 were upregulated in the lesional skin and six were downregulated (Additional file 7: Table S5). Several of these miRNAs have been shown to be differentially expressed in psoriatic skin in previous studies and are highlighted in Fig. 6c.

\section{Investigation of potential interactions between circRNAs and miRNAs}

To analyze potential interactions between miRNAs and circRNAs in psoriasis, we considered only the high abundance circRNAs and miRNAs as the quantitative data for these are much more reliable than for circRNAs and miRNAs supported by very few reads. We first predicted miRNA binding sites in each of the individual circRNAs and multiplied the number of sites with the change in expression ( $\triangle R P M$ values) between lesionaland non-lesional skin for each corresponding circRNA. The sum of the $\triangle R P M$ values for each miRNA binding site was then plotted against the fold changes (Fig. 7a) and absolute changes (Fig. 7b) in miRNA expression levels between lesional- and non-lesional skin, respectively. For both of these analyses, we did not observe any correlation between the loss of miRNA binding sites in the circRNAs and differences in miRNA expression levels between lesional and non-lesional skin. The best candidate for a miRNA that was differentially expressed as a result of changes in the circRNAome was miR203a-3p (Fig. 7) as many miR-203a-3p binding sites were present in downregulated circRNAs and miR-203a-3p was among the most upregulated miRNAs in lesional skin. However, this does not seem to reflect a general interplay between circRNAs and miRNAs in psoriasis.

\section{Discussion}

In this study, we present the first sequencing-based cata$\log$ of circRNA expression in lesional- and non-lesional skin from patients diagnosed with psoriasis vulgaris. Our data show that the circRNAome is extensively downregulated in lesional skin. The RNA-seq data were confirmed using an enzyme-free method [43], which is not subject to PCR bias and potential artifacts related to reverse transcription [42-44], and could be extended to another patient cohort. Also, we observed an overall downregulation of circRNAs when comparing lesional skin to normal skin from healthy individuals.

The circRNAome was mainly downregulated in the epidermis of the skin, an effect that could not be explained by differential expression of known circRNA biogenesis factors nor by differences in the number of infiltrating lymphocytes. We did observe that $A D A R$ was expressed at significantly higher levels in the lesional skin, but there was no tendency for circRNAs with IAEs in the introns flanking their BSJs to be more downregulated than circRNAs without, which argues against upregulation of $A D A R$ as the main mechanism responsible for downregulation of the circRNAome in psoriasis. In addition, despite being statistically significant, the difference in $A D A R$ expression was less than two fold. Thus, a more likely explanation for the altered circRNA levels may involve the higher proliferation and turnover rates of the keratinocytes in lesional skin, and the fact that those keratinocytes are less likely to become terminally differentiated. It has previously been observed that circRNAs are upregulated during differentiation $[18,25]$ and that higher levels of these molecules are found in non-proliferating cells [48]. In particular, circRNA molecules have very long half-lives in cells due to their slow turnover rates [9] and the relative inefficiency of the backsplicing reaction required to form most circRNAs imply that they generally need longer time to build up to steady-state levels. Thus, we propose that the less differentiated and highly proliferative keratinocytes in the lesional psoriatic skin prevent the highly stable circRNA molecules from building up to the same expression levels found in the non-lesional skin cells. In support of this hypothesis, we observed that most of the circRNA expression changes were independent of expression changes of their respective linear host genes. In other words, less active promoters and enhancers may only explain a minor part of the observed downregulation of the circRNAome. In addition, when analyzing different classes of linear RNA, including miRNAs, mRNAs, lincRNAs, antisense RNAs, and snoRNAs, we did not observe an overall downregulation in lesional skin relative to non-lesional skin.

Interestingly, a recent study has shown that circRNAs are downregulated as a class upon viral infection [49]. Using genome-wide siRNA screening targeting all unique human genes and an efficient circRNA expression reporter, this 


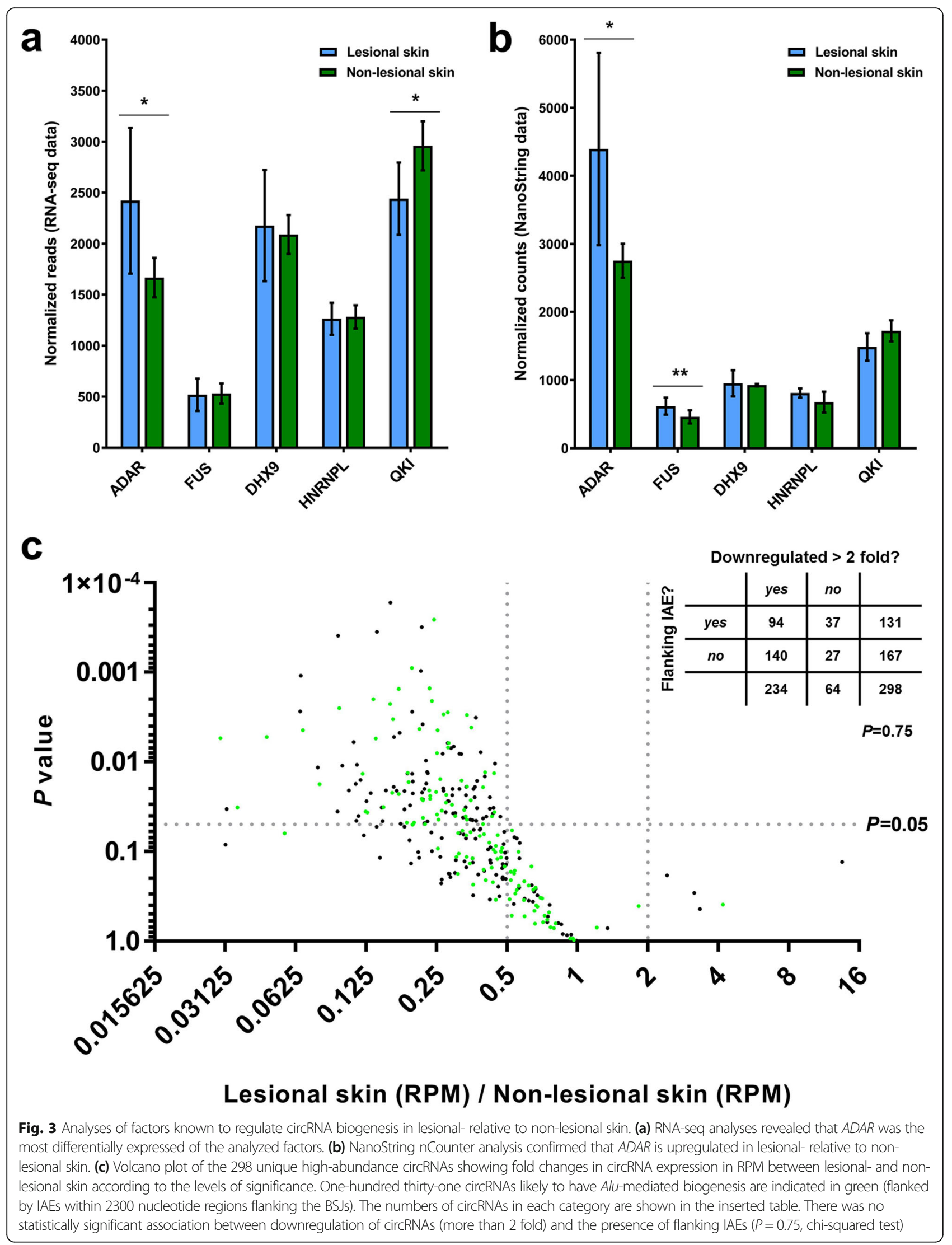




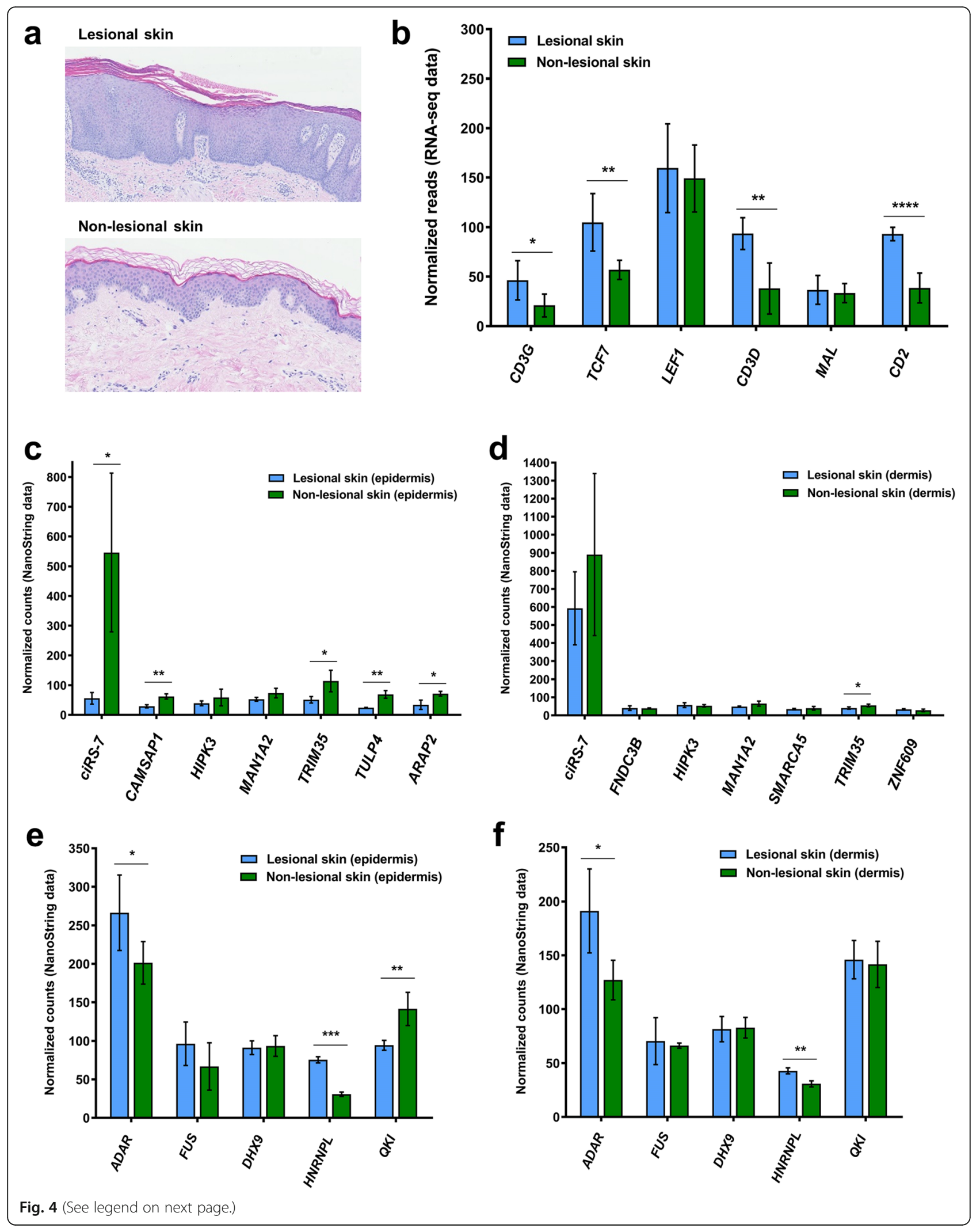


(See figure on previous page.)

Fig. 4 The downregulation of circRNAs, mainly observed in the epidermis, is unlikely to be caused by infiltrating lymphocytes. (a) Representative H\&E staining of lesional- and non-lesional skin samples showing a relative abundance of lymphocytes in the dermis of the lesional skin. (b) RNAseq data showed that T-cell specific genes were expressed at higher levels in the lesional skin- relative to non-lesional skin. (c-d) Following microdissection of the epidermis and NanoString nCounter analysis, five of seven circRNAs were shown to be significantly downregulated in the epidermis of the lesional skin (c), while this only applied to one of seven circRNAs analyzed in the dermis (d). (e-f) ADAR proved to be significantly upregulated both in the epidermis (e) and the dermis (f) of the lesional skin

study identified the immune factors NF90/NF110 as key regulators in circRNA biogenesis. The gene encoding these factors (ILF3) was not differentially expressed in our RNAseq data, which was expected since the immune pathways activated in psoriasis are different from those activated upon viral infection. However, we cannot exclude that other yet unknown factors could play a role in the downregulation of circRNAs in psoriasis. For instance, a recent study has provided evidence that endogenous circRNAs tend to form 16-26 bp imperfect RNA duplexes and can act as inhibitors of double-stranded RNA (dsRNA)-activated protein kinase (PKR), which is related to innate immunity. Consequently, upon poly(I:C) stimulation or viral infection, circRNAs are globally degraded by an intracellular RNase, RNase L, a process essential for PKR activation in early cellular innate immune responses. Moreover, increased PKR phosphorylation and circRNA downregulation were observed in peripheral blood mononuclear cells
(PBMCs) derived from patients with systemic lupus erythematosus (SLE) [50].

Deregulation of miRNA levels has been implicated in the pathogenesis of psoriasis and numerous studies have indicated that circRNAs may function as efficient inhibitors of miRNA activity $[10,24,30,51]$. Therefore, we hypothesized that loss of miRNA binding sites, as a consequence of the downregulated circRNAome, could explain some of the changes in miRNA expression observed in psoriasis. To test this hypothesis, we profiled the expression of many miRNAs in the skin biopsies and quantified the number of individual miRNA binding sites in each of the high abundance circRNA. We were unable to find any correlations between the loss of miRNA binding sites on the circRNAs and differences in miRNA expression levels between lesional and nonlesional skin. However, these analyses are limited by the possibility that a subset of the multi-exonic circRNAs

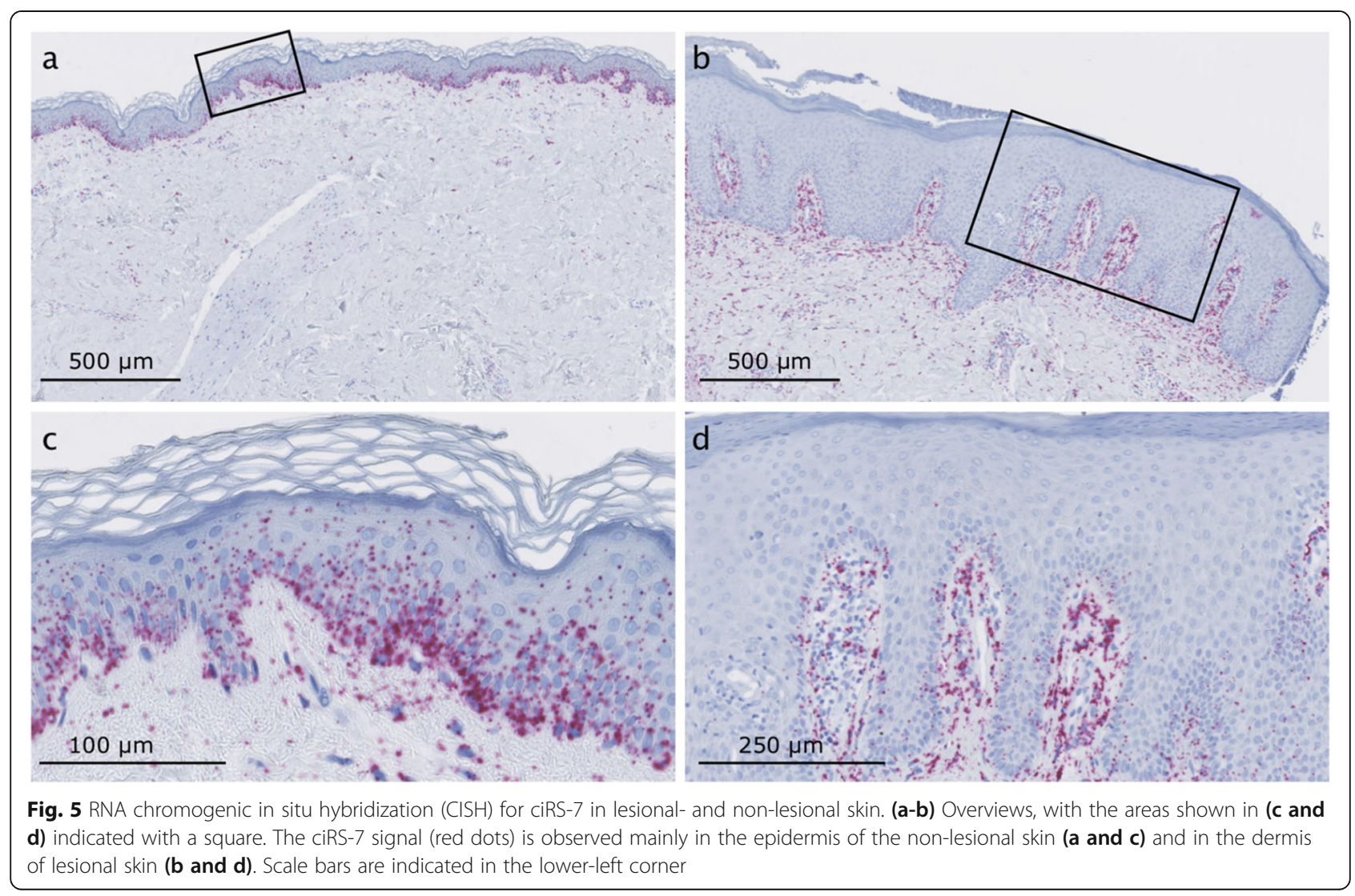




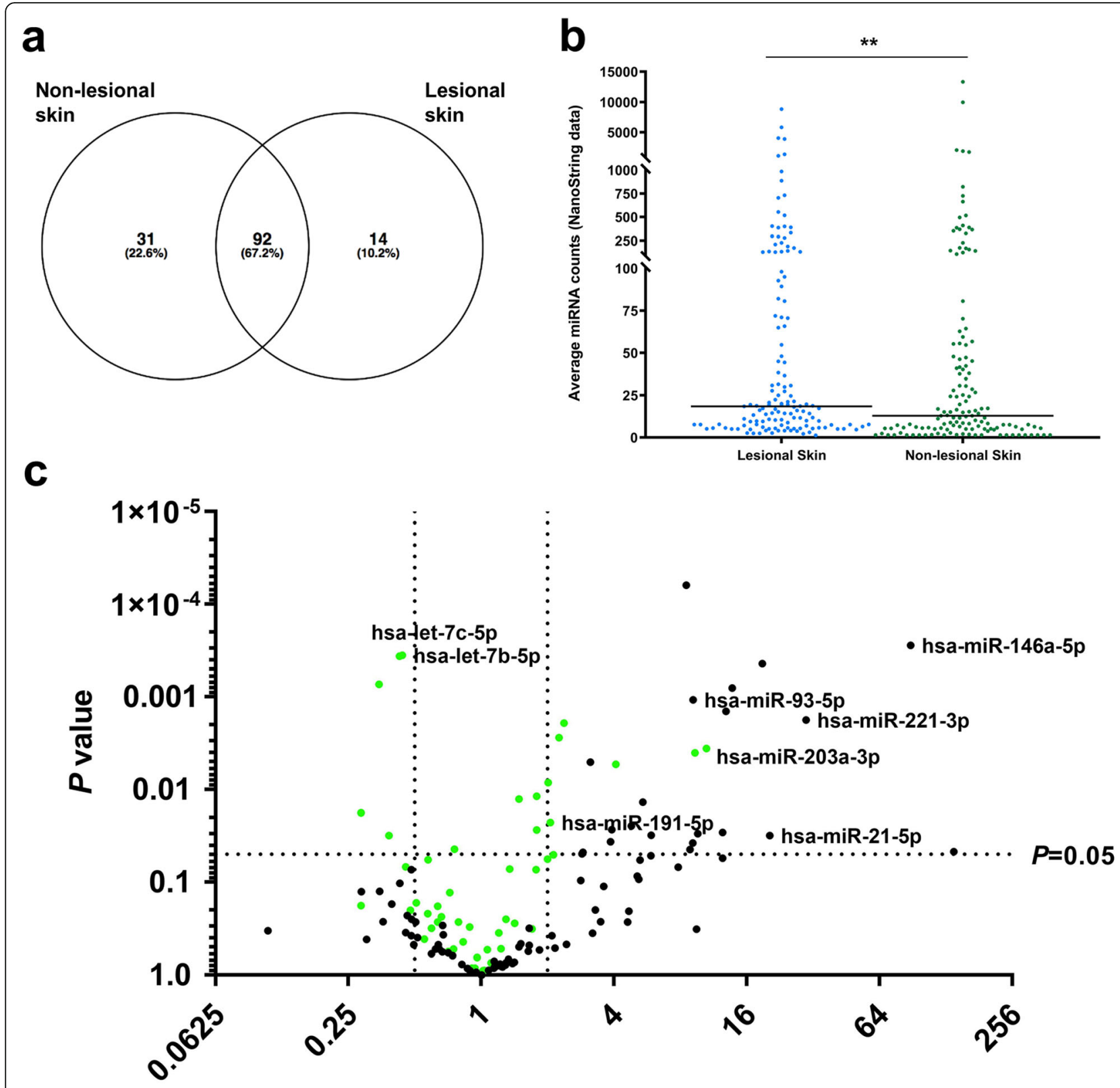

Lesional Skin (counts)/ Non-lesional Skin (counts)

Fig. 6 Characterization of miRNAs in lesional skin- and non-lesional skin using NanoString nCounter analysis. (a) Venn diagram illustrating the overlap between the miRNAs detected in lesional- and non-lesional skin. (b) The average expression of the 137 high abundance miRNAs is slightly lower in lesional- relative to non-lesional skin. (c) Volcano plot of the 137 high abundance miRNAs, with the top 50 most abundant miRNAs indicated in green along with the names of miRNAs previously shown to be differentially expressed in psoriasis

analyzed could be subject to alternative splicing or intron retention events. Together, our observations may suggest that the global reduction in circRNA expression in lesional skin is more likely to be a consequence than a cause of the disease. However, this does not necessarily imply that individual circRNAs may not be directly involved in psoriasis pathogenesis.
In fact, a recent study argues that circRNAs are directly involved in the development of the disease [52]. The authors of this study performed RNA-seq of stem cells isolated from lesional psoriatic skin and normal skin from healthy individuals. Compared to our study, the patients had a wider range of disease severity (PASI scores between 3.0 and 43.8), and the stem cells were cultured for five generations before 
a

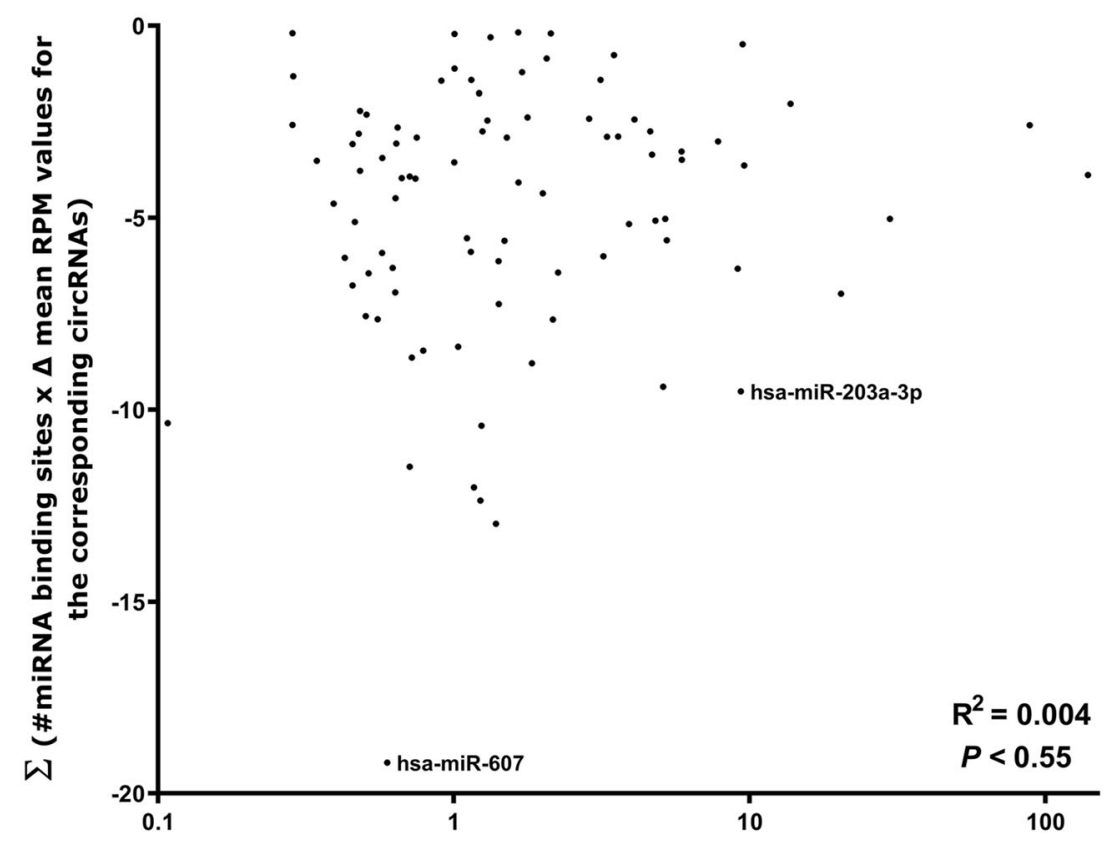

mean miRNA counts (lesional) / mean miRNA counts (non-lesional)

b

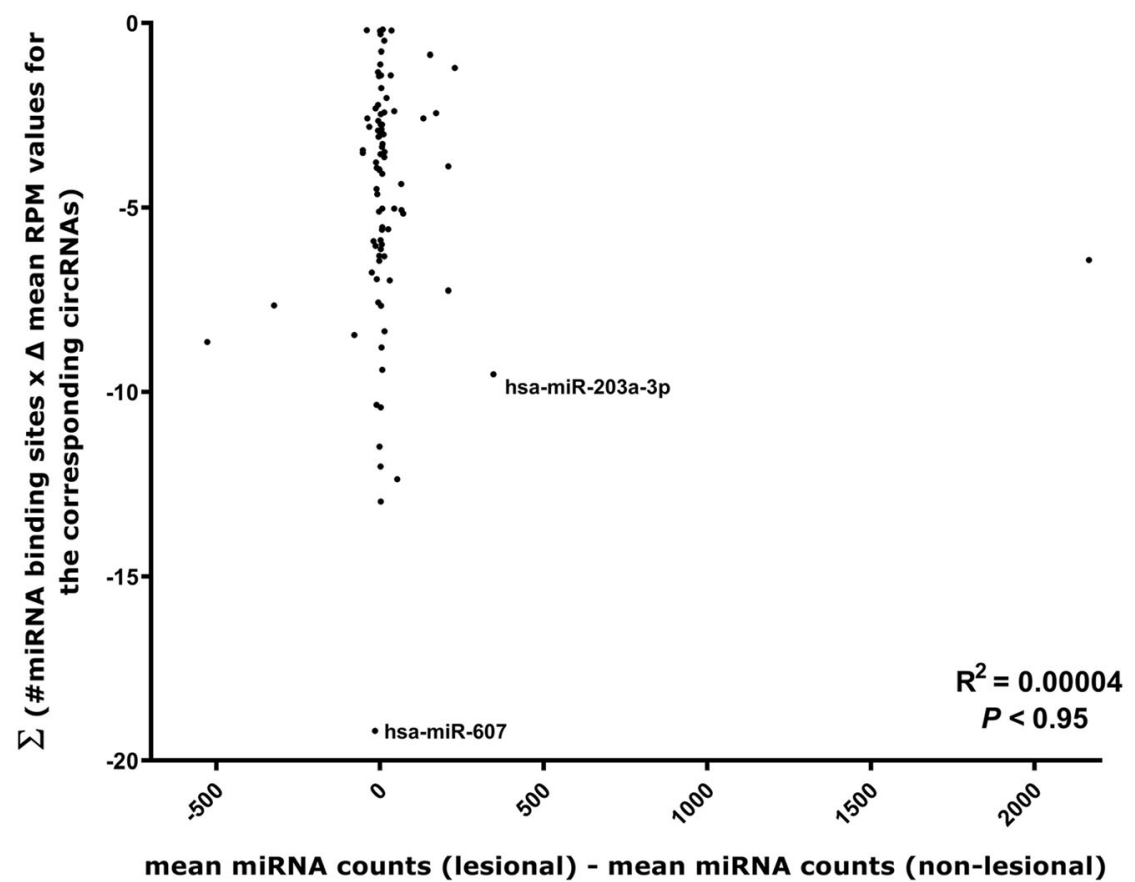

Fig. 7 Changes in the amounts of available miRNA binding sites present on circRNAs do not correlate with changes in miRNA expression levels in lesional- relative to non-lesional skin. (a-b) For each miRNA, the sum of the number of binding sites for that particular miRNA was multiplied with the mean circRNA RPM (lesional) - mean circRNA RPM (non-lesional) for the circRNAs harboring binding sites for that particular miRNA. These values were plotted against either the fold change (mean miRNA counts (lesional)/mean miRNA counts (non-lesional)) for each high abundance miRNA with at least one binding site present on the high abundance circRNAs (a) or the absolute difference in expression level (mean miRNA counts (lesional) mean miRNA counts (non-lesional)) for each high abundance miRNA with at least one binding site present on the high abundance circRNAs (b) 
RNA-seq analyses. The bioinformatics algorithm CIRI was used for circRNA quantification, which we have previously shown to have a low accuracy for circRNA detection [53]. That could potentially explain why more than half of the 6000 circRNAs detected are not present in circBase. The authors did not validate the circular nature of any of these potentially novel circRNAs by assessing their resistance to degradation by RNase R and no comparisons to other bioinformatics algorithms were performed [52]. In contrast to our study, the authors found many more upregulated- than downregulated circRNAs and proceeded to functionally study a potentially novel circRNA derived from chr2: 206992521|206,994,966. This circRNA was predicted to interact with several miRNAs, which were thought to regulate STAT3, STAT4, and IL2RB. However, no experimental evidence was provided in support of this [52]. We did not find this particular circRNA among the more than 3000 unique circRNAs detected in the lesional- and non-lesional skin biopsies and could, therefore, not examine if it is differentially expressed in our samples.

Another recent study profiled circRNA expression in three psoriatic lesions and three normal healthy skin tissues [54]. The RNA from these samples was amplified and then subjected to microarray analysis. The authors found almost 5000 differentially expressed circRNAs of which the majority were upregulated in psoriasis. Six differentially expressed circRNAs were then selected for verification by RT-qPCR, but only one of these, hsa_circ_0061012, could be validated [54]. Again, we did not detect this circRNA using RNA-seq and could, therefore, not examine if it is differentially expressed in our samples.

To the best of our knowledge, our study is the first to use the NanoString nCounter technology to profile miRNA expression in psoriasis and, in accordance with earlier reports, we found that miR-146a, miR-21, miR-203, miR-221, miR-155, and miR-223 were significantly upregulated, and that hsa-let-7b-5p, hsa-let-7c-5p, and miR-125b were significantly downregulated in lesional- relative to non-lesional skin $[4,5]$. Additionally, we identified several miRNAs that have not previously been associated with psoriasis. For instance, miR-200c-3p, known to enhance the invasive capacity of human squamous cell carcinoma [55], miR-191-5p, abnormally expressed in several cancers and various other diseases [56], and hsa-miR-93-5p, promoting cancer cell proliferation through inhibiting $L K B 1$ in lung adenocarcinoma [57], were all significantly upregulated in the lesional skin.

\section{Conclusions}

In this study, we have found a global reduction of circRNA expression levels in lesional- relative to non-lesional skin from patients diagnosed with psoriasis. This phenomenon was mainly restricted to the epidermis and could not be explained by expression changes in factors known to affect
circRNA biogenesis nor by differences in the number of lymphocytes in the samples. Instead, we suggest that the downregulated circRNAome in lesional skin may be related to a passive dilution of the circRNAs caused by the high proliferation- and turnover rates of the keratinocytes in the epidermis of the skin. It is too early to say whether the altered circRNA expression in psoriasis is a cause or consequence of the disease, however, our data do not support an active role for circRNAs in psoriasis pathogenesis via alteration of miRNA expression levels.

\section{Supplementary information}

Supplementary information accompanies this paper at https://doi.org/10. 1186/s12920-019-0616-2.

Additional file 1: Figure S1. (a-f) Scatter plots showing the average expression in reads per million (RPM) of the 298 unique high-abundance circRNAs in lesional- and non-lesional skin for each of the individual patients. The bars represent means.

Additional file 2: Figure S2. (a) Volcano plot of the top 298 most abundant mRNAs showing fold changes in mRNA expression between lesional- and non-lesional skin according to the levels of significance. Several genes known to be upregulated in psoriasis are indicated. (b) Volcano plot of the top 298 most abundant lincRNAs showing fold changes in lincRNA expression between lesional- and non-lesional skin according to the levels of significance. (c) Volcano plot of the top 298 most abundant antisense RNAs showing fold changes in antisense RNA expression between lesional- and non-lesional skin according to the levels of significance. (d) Volcano plot of the top 298 most abundant snoRNAs showing fold changes in snoRNA expression between lesional- and non-lesional skin according to the levels of significance.

Additional file 3: Figure S3. (a) Volcano plot of the top 50 most abundant circRNAs showing fold changes in circRNA expression in counts between lesional- and non-lesional skin from the first cohort according to the levels of significance. (b) Volcano plot of the top 50 most abundant circRNAs showing fold changes in circRNA expression in counts between lesional skin and healthy control skin according to the levels of significance. (c) Volcano plot of the top 50 most abundant circRNAs showing fold changes in circRNA expression in counts between lesionaland non-lesional skin from a second cohort of 13 patients according to the levels of significance. All analyses were performed using our custom NanoString nCounter panel.

Additional file 4: Figure S4. NanoString nCounter analysis confirmed that $A D A R$ is upregulated in lesional- relative to non-lesional skin samples from a second cohort of 13 patients.

Additional file 5: Figure S5. Volcano plot of the 298 unique highabundance circRNAs showing fold changes in circRNA expression in RPM between lesional- and non-lesional skin according to the levels of significance. Two-hundred thirty-three circRNAs likely to have Alu-mediated biogenesis are indicated in green (flanked by IAEs from the same subfamily within 10,000 nucleotide regions flanking the BSJs). Numbers of cirCRNA in each category are shown in the inserted table. There was no statistically significant association between downregulation of circRNAs (more than 2 fold) and the presence of flanking IAEs from the same subfamily ( $P=0.60$, chi-squared test).)

Additional file 6: Figure S6. (a-f) Scatter plots showing the average expression in counts of the 137 unique high-abundance miRNAs in lesional- and non-lesional skin for each of the individual patients. The bars represent means.

Additional file 7: Table S1. Custom CodeSet of capture- and reporter probes designed to target regions of $100 \mathrm{bp}$ overlaying the BSJs of the top 50 most abundant circRNAs in the entire dataset. Table S2. The 128 high abundance circRNAs detected in the lesional skin biopsies listed according to average RPM. Table S3. The 285 high abundance circRNAs 
detected in the non-lesional skin biopsies listed according to average RPM. Table S4. The 148 circRNAs significantly downregulated in the lesional- relative to non-lesional skin. Table $\mathbf{S 5}$. The 37 significantly differentially expressed miRNAs among the 137 high abundance miRs.

\section{Abbreviations}

ADAR1: Adenosine deaminase acting on RNA; bp: Base pairs; BSJ: Backsplice junction; circRNA: Circular RNA; ciRS-7: circular RNA sponge for miR-7; CISH: Chromogenic in situ hybridization; CTLR: Circular-to-linear ratio; FFPE : Formalin-fixed paraffin-embedded; FUS: Fused in Sarcoma RNA-binding protein; HNRNPL: Heterogeneous nuclear ribonucleoprotein L; IAE: Inverted ALU elements; lincRNA: Long intergenic noncoding RNA; IncRNA: Long noncoding RNA; miR-7: microRNA 7; miRNA: MicroRNA; mRNA: Messenger RNA; nt: Nucleotides; PASI: Psoriasis Area Severity Index; PCR: Polymerase chain reaction; QKI: Quaking; RNase R: Ribonuclease R; RNA-seq: RNA sequencing; RPM: Reads per million; rRNA: Ribosomal RNA: RT: Reverse transcription; RTQPCR: Quantitative reverse transcription PCR; snoRNAs: Small nucleolar RNA

\section{Acknowledgements}

We thank Birgit MacDonald, Malene Hykkelbjerg Nielsen and Birgit Roed Sørensen for their outstanding technical assistance and Anne Færch Nielsen for a thorough and critical reading of the manuscript.

\section{Authors' contributions}

LSK, LM, LI, CJ and JK conceived the study. CJ and LI collected the clinical samples used. LM and LSK carried out the laboratory work related to RNAseq and NanoString nCounter analyses. TLA carried out the RNA CISH analysis. HH carried out microdissections and H\&E stainings. TLHO and TBH carried out the bioinformatics analysis related to IAE in BSJ flanking regions. MTV and LSK performed the bioinformatics analyses related to RNA-seq experiments. LM and LSK analyzed all data and prepared the figures and Tables. JK and LSK provided reagents and materials. LSK wrote the draft version of the manuscript, which was revised for important intellectual content and approved by all authors.

\section{Funding}

This work was supported by the European Union's Horizon 2020 research and innovation programme under the Marie Skłodowska-Curie grant agreement No 721890, by the Villum Foundation and Independent Research Fund Denmark | Natural Sciences, and LSK is supported by a grant from the Carlsberg Foundation (CF16-0087). The funding bodies provided financial support but had no other role in the design of the study, data collection, analysis, and interpretation of data, decision to publish, or preparation of the manuscript.

\section{Availability of data and materials}

The datasets supporting the conclusions of this article are included within the article and its supplementary files. Our raw data cannot be submitted to publicly available databases because the patients were not consented for sharing their raw data, which can potentially identify the individuals, but are available from the corresponding author on reasonable request.

\section{Ethics approval and consent to participate}

This study was conducted according to the Declaration of Helsinki Principles. Written informed consent was obtained from each patient and permissions from the Regional Ethical Committee of Region Midtjylland, Denmark (M20090102) and the Danish National Committee on Health Research Ethics (1807975) were granted.

\section{Consent for publication}

$$
\text { Not applicable. }
$$

\section{Competing interests}

The authors declare no competing financial interests.

\section{Author details}

'Department of Molecular Biology and Genetics (MBG), Aarhus University, DK-8000 Aarhus, Denmark. ${ }^{2}$ Interdisciplinary Nanoscience Center (iNANO), Aarhus University, DK-8000 Aarhus, Denmark. ${ }^{3}$ Department of Molecular Medicine (MOMA), Aarhus University Hospital, DK-8200 Aarhus, Denmark.
${ }^{4}$ Clinical Cell Biology, Research Unit of Pathology, Department of Clinical Research, University of Southern Denmark, DK-5000 Odense, Denmark. ${ }^{5}$ Department of Clinical Pathology, Vejle Hospital, DK-7100 Vejle, Denmark. ${ }^{6}$ Department of Dermatology, Aarhus University Hospital, DK-8000 Aarhus, Denmark.

Received: 10 April 2019 Accepted: 8 November 2019

Published online: 27 November 2019

\section{References}

1. Lebwohl M. Psoriasis. Lancet. 2003;361:1197-204.

2. Lowes MA, Bowcock AM, Krueger JG. Pathogenesis and therapy of psoriasis. Nature. 2007:445:866-73.

3. Nomura I, Gao B, Boguniewicz M, Darst MA, Travers JB, Leung DY. Distinct patterns of gene expression in the skin lesions of atopic dermatitis and psoriasis: a gene microarray analysis. J Allergy Clin Immunol. 2003;112:1195-202.

4. Sonkoly $E$, Wei $T$, Janson PC, Saaf A, Lundeberg L, Tengvall-Linder M, Norstedt G, Alenius H, Homey B, Scheynius A, et al. MicroRNAs: novel regulators involved in the pathogenesis of psoriasis? PLoS One. 2007;2:e610.

5. Zibert JR, Lovendorf MB, Litman T, Olsen J, Kaczkowski B, Skov L. MicroRNAs and potential target interactions in psoriasis. J Dermatol Sci. 2010;58:177-85.

6. Ahn R, Gupta R, Lai K, Chopra N, Arron ST, Liao W. Network analysis of psoriasis reveals biological pathways and roles for coding and long noncoding RNAs. BMC Genomics. 2016;17:841.

7. Gupta R, Ahn R, Lai K, Mullins E, Debbaneh M, Dimon M, Arron S, Liao W. Landscape of long noncoding RNAs in psoriatic and healthy skin. J Invest Dermatol. 2016;136:603-9.

8. Tsoi LC, lyer MK, Stuart PE, Swindell WR, Gudjonsson JE, Tejasvi T, Sarkar MK, Li B, Ding J, Voorhees JJ, et al. Analysis of long non-coding RNAs highlights tissue-specific expression patterns and epigenetic profiles in normal and psoriatic skin. Genome Biol. 2015;16:24.

9. Jeck WR, Sorrentino JA, Wang K, Slevin MK, Burd CE, Liu J, Marzluff WF, Sharpless NE. Circular RNAs are abundant, conserved, and associated with ALU repeats. Rna. 2013;19:141-57.

10. Memczak S, Jens M, Elefsinioti A, Torti F, Krueger J, Rybak A, Maier L, Mackowiak SD, Gregersen LH, Munschauer M, et al. Circular RNAs are a large class of animal RNAs with regulatory potency. Nature. 2013;495:333-8.

11. Salzman J, Gawad C, Wang PL, Lacayo N, Brown PO. Circular RNAs are the predominant transcript isoform from hundreds of human genes in diverse cell types. PLoS One. 2012;7:e30733.

12. Glazar P, Papavasileiou P. Rajewsky N: circBase: a database for circular RNAs. RNA. 2014;20:1666-70.

13. Dong R, Ma XK, Li GW, Yang L. CIRCpedia v2: an updated database for comprehensive circular RNA annotation and expression comparison. Genomics Proteomics Bioinform. 2018;16:226-33.

14. Kristensen LS, Hansen TB, Veno MT, Kjems J. Circular RNAs in cancer: opportunities and challenges in the field. Oncogene. 2018;37:555-65.

15. Ebbesen KK, Kjems J, Hansen TB. Circular RNAs: identification, biogenesis and function. Biochim Biophys Acta. 1859:2016:163-8.

16. Ivanov A, Memczak S, Wyler E, Torti F, Porath HT, Orejuela MR, Piechotta M, Levanon EY, Landthaler M, Dieterich C, Rajewsky N. Analysis of intron sequences reveals hallmarks of circular RNA biogenesis in animals. Cell Rep. 2015;10:170-7.

17. Kristensen LS, Andersen MS, Stagsted LW, Ebbesen KK, Hansen TB, Kjems J. The biogenesis, biology and characterization of circular RNAs. Nat Rev Genet. 2019;20:675-91.

18. Rybak-Wolf A, Stottmeister C, Glazar P, Jens M, Pino N, Giusti S, Hanan M, Behm M, Bartok O, Ashwal-Fluss R, et al. Circular RNAs in the mammalian brain are highly abundant, conserved, and dynamically expressed. Mol Cell. 2015:58:870-85

19. Yoshimoto R, Rahimi K, Hansen T, Kjems J, Mayeda A: Biosynthesis of Circular RNA ciRS-7/CDR1as Is Mediated by Mammalian-Wide Interspersed Repeats (MIRs). bioRxiv 2019:411231.

20. Aktas T, Avsar Ilik I, Maticzka D, Bhardwaj V, Pessoa Rodrigues C, Mittler G, Manke T, Backofen R, Akhtar A. DHX9 suppresses RNA processing defects originating from the Alu invasion of the human genome. Nature. 2017:544:115-9.

21. Fei T, Chen Y, Xiao T, Li W, Cato L, Zhang P, Cotter MB, Bowden M, Lis RT, Zhao SG, et al. Genome-wide CRISPR screen identifies HNRNPL as a prostate 
cancer dependency regulating RNA splicing. Proc Natl Acad Sci U S A. 2017; 114:E5207-e5215.

22. Conn SJ, Pillman KA, Toubia J, Conn VM, Salmanidis M, Phillips CA, Roslan S, Schreiber AW, Gregory PA, Goodall GJ. The RNA binding protein quaking regulates formation of circRNAs. Cell. 2015;160:1125-34.

23. Errichelli L, Dini Modigliani S, Laneve P, Colantoni A, Legnini I, Capauto D, Rosa A, De Santis R, Scarfo R, Peruzzi G, et al. FUS affects circular RNA expression in murine embryonic stem cell-derived motor neurons. Nat Commun. 2017;8:14741.

24. Hansen $T B$, Jensen $T I$, Clausen BH, Bramsen JB, Finsen B, Damgaard CK, Kjems J. Natural RNA circles function as efficient microRNA sponges. Nature. 2013:495:384-8.

25. Kristensen LS, Okholm TLH, Veno MT, Kjems J. Circular RNAs are abundantly expressed and upregulated during human epidermal stem cell differentiation. RNA Biol. 2018:15:280-91.

26. Yu CY, Li TC, Wu YY, Yeh CH, Chiang W, Chuang CY, Kuo HC. The circular RNA circBIRC6 participates in the molecular circuitry controlling human pluripotency. Nat Commun. 2017;8:1149.

27. Liang Y, Song X, Li Y, Su P, Han D, Ma T, Guo R, Chen B, Zhao W, Sang Y, et al. CircKDM4C suppresses tumor progression and attenuates doxorubicin resistance by regulating miR-548p/PBLD axis in breast cancer. Oncogene. 2019;38:6850-66.

28. Verduci L, Ferraiuolo M, Sacconi A, Ganci F, Vitale J, Colombo T, Paci P, Strano S, Macino G, Rajewsky N, Blandino G. The oncogenic role of circPVT1 in head and neck squamous cell carcinoma is mediated through the mutant p53/YAP/TEAD transcription-competent complex. Genome Biol. 2017:18:237.

29. Kleaveland B, Shi CY, Stefano J, Bartel DP. A Network of Noncoding Regulatory RNAs Acts in the Mammalian Brain. Cell. 2018;174:350-362.e317.

30. Piwecka M, Glazar P, Hernandez-Miranda LR, Memczak S, Wolf SA, RybakWolf A, Filipchyk A, Klironomos F, Cerda Jara CA, Fenske P, et al. Loss of a mammalian circular RNA locus causes miRNA deregulation and affects brain function. Sci. 2017;357.

31. Guo JU, Agarwal V, Guo H, Bartel DP. Expanded identification and characterization of mammalian circular RNAs. Genome Biol. 2014;15:409.

32. Thomson DW, Dinger ME. Endogenous microRNA sponges: evidence and controversy. Nat Rev Genet. 2016;17:272-83.

33. Veno MT, Hansen TB, Veno ST, Clausen BH, Grebing M, Finsen B, Holm IE, Kjems J. Spatio-temporal regulation of circular RNA expression during porcine embryonic brain development. Genome Biol. 2015;16:245.

34. Zhang XO, Wang HB, Zhang Y, Lu X, Chen LL, Yang L. Complementary sequence-mediated exon circularization. Cell. 2014;159:134-47.

35. Liao Y, Smyth GK, Shi W. featureCounts: an efficient general purpose program for assigning sequence reads to genomic features. Bioinform. 2014;30:923-30

36. Stagsted LV, Nielsen KM, Daugaard I, Hansen TB. Noncoding AUG circRNAs constitute an abundant and conserved subclass of circles. Life Sci Alliance. 2019;2.

37. Okholm TLH, Nielsen MM, Hamilton MP, Christensen L-L, Vang S, Hedegaard J, Hansen TB, Kjems J, Dyrskjøt L, Pedersen JS: Circular RNA expression is abundant and correlated to aggressiveness in early-stage bladder cance npj Genomic Medicine 2017, 2:36.

38. Allen D, Winters E, Kenna PF, Humphries P, Farrar GJ. Reference gene selection for real-time rtPCR in human epidermal keratinocytes. J Dermatol Sci. 2008:49:217-25.

39. Andersen $\mathrm{CL}$, Jensen $\mathrm{JL}$, Orntoft TF. Normalization of real-time quantitative reverse transcription-PCR data: a model-based variance estimation approach to identify genes suited for normalization, applied to bladder and colon cancer data sets. Cancer Res. 2004:64:5245-50.

40. Bartel DP. MicroRNAs: target recognition and regulatory functions. Cell. 2009;136:215-33.

41. Lassen NE, Andersen TL, Ploen GG, Soe K, Hauge EM, Harving S, Eschen GET, Delaisse JM. Coupling of bone Resorption and formation in real time: new knowledge gained from human Haversian BMUs. J Bone Miner Res. 2017;32:1395-405.

42. Chen D-F, Zhang L-J, Tan K, Jing Q. Application of droplet digital PCR in quantitative detection of the cell-free circulating circRNAs. Biotechnol \& Biotechnol Equip. 2017:1-8.

43. Dahl M, Daugaard I, Andersen MS, Hansen TB, Gronbaek K, Kjems J, Kristensen LS. Enzyme-free digital counting of endogenous circular RNA molecules in B-cell malignancies. Lab Invest. 2018;98:1657-69.
44. Szabo L, Salzman J. Detecting circular RNAs: bioinformatic and experimental challenges. Nat Rev Genet. 2016:17:679-92.

45. Schon MP, Erpenbeck L. The Interleukin-23/Interleukin-17 Axis links adaptive and innate immunity in psoriasis. Front Immunol. 2018;9:1323.

46. Morganroth GS, Chan LS, Weinstein GD, Voorhees JJ, Cooper KD. Proliferating cells in psoriatic dermis are comprised primarily of $\mathrm{T}$ cells, endothelial cells, and factor XIlla+ perivascular dendritic cells. J Invest Dermatol. 1991;96:333-40.

47. Palmer C, Diehn M, Alizadeh AA, Brown PO. Cell-type specific gene expression profiles of leukocytes in human peripheral blood. BMC Genomics. 2006;7:115.

48. Bachmayr-Heyda A, Reiner AT, Auer K, Sukhbaatar N, Aust S, BachleitnerHofmann T, Mesteri I, Grunt TW, Zeillinger R, Pils D. Correlation of circular RNA abundance with proliferation--exemplified with colorectal and ovarian cancer, idiopathic lung fibrosis, and normal human tissues. Sci Rep. 2015;5:8057.

49. Li X, Liu CX, Xue W, Zhang Y, Jiang S, Yin QF, Wei J, Yao RW, Yang L, Chen LL. Coordinated circRNA Biogenesis and Function with NF90/NF110 in Viral Infection. Mol Cell. 2017;67:214-227.e217.

50. Liu CX, Li X, Nan F, Jiang S, Gao X, Guo SK, Xue W, Cui Y, Dong K, Ding H, et al. Structure and Degradation of Circular RNAs Regulate PKR Activation in Innate Immunity. Cell. 2019;177:865-880.e821.

51. Zheng Q, Bao C, Guo W, Li S, Chen J, Chen B, Luo Y, Lyu D, Li Y, Shi G, et al. Circular RNA profiling reveals an abundant circHIPK3 that regulates cell growth by sponging multiple miRNAs. Nat Commun. 2016;7:11215.

52. Liu R, Chang W, Li J, Cheng Y, Dang E, Yang X, Wang Q, Wang G, Li X, Zhang K. Mesenchymal stem cells in psoriatic lesions affect the skin microenvironment through circular RNA. Experimental Dermatology. 2019; 28:292-9.

53. Hansen TB, Veno MT, Damgaard CK, Kjems J. Comparison of circular RNA prediction tools. Nucleic Acids Res. 2016;44:e58.

54. Qiao M, Ding J, Yan J, Li R, Jiao J, Sun Q. Circular RNA expression profile and analysis of their potential function in psoriasis. Cell Physiol Biochem. 2018:50:15-27.

55. Kawakubo-Yasukochi T, Morioka M, Hazekawa M, Yasukochi A, Nishinakagawa T, Ono K, Kawano S, Nakamura S, Nakashima M. miR-200c-3p spreads invasive capacity in human oral squamous cell carcinoma microenvironment. Mol Carcinog. 2018;57:295-302.

56. Nagpal N, Kulshreshtha R. miR-191: an emerging player in disease biology. Front Genet. 2014;5:99

57. Du L, Zhao Z, Ma X, Hsiao TH, Chen Y, Young E, Suraokar M, Wistuba I, Minna JD, Pertsemlidis A. miR-93-directed downregulation of DAB2 defines a novel oncogenic pathway in lung cancer. Oncogene. 2014;33:4307-15.

\section{Publisher's Note}

Springer Nature remains neutral with regard to jurisdictional claims in published maps and institutional affiliations.

Ready to submit your research? Choose BMC and benefit from

- fast, convenient online submission

- thorough peer review by experienced researchers in your field

- rapid publication on acceptance

- support for research data, including large and complex data types

- gold Open Access which fosters wider collaboration and increased citations

- maximum visibility for your research: over $100 \mathrm{M}$ website views per year

At $\mathrm{BMC}$, research is always in progress.

Learn more biomedcentral.com/submissions 